\title{
Las funciones pragmáticas y la posición del sujeto en español
}

\section{Introducción}

El estudio de las funciones pragmáticas cuenta ya con una sólida tradición en la lingüística, y en particular ha interesado a la investigación, que trabaja desde enfoques funcionales en el estudio de la gramática (Dik 1989, 1997), así como los que se han interesado por describir la estructura y organización del texto y el discurso (van Dijk 1981, Goutsos 1997). De un modo más específico y detallado, la noción de tema (o tema-tópico) se ha tratado desde la sintaxis (Gundel 1985, Cadiot 1992, Lambrecht 1994) y el discurso oral (Maynard 1980, Chafe 1994, Morris 1988, Hidalgo Downing 2003). En estudios más recientes, se ha explorado el impacto de la dimensión temática en la estructura discursiva oral, a partir de un tipo de discurso oral planificado, como es el discurso político (Cortés Rodríguez e Hidalgo Downing 2015) así como en las posibilidades de anotación de corpus (Dipper et al. 2007, Hidalgo Downing y Downing 2012).

Por otro lado, la posición del sujeto en español ha recibido extensa atención en la lingüística española, y tanto desde enfoques formales como funcionales se ha tratado de describir la variación en las posiciones sintácticas del sujeto, así como de los factores que pueden determinar tal posición. Desde estudios tradicionales sobre el orden de palabras en español (Contreras 1978), un rasgo común es el de destacar que la posición del sujeto no está condicionada por un único factor, sino que recoge la confluencia de factores semánticos, sintácticos y pragmáticos (Fernández Soriano 1993), como el tipo de verbo (ergativo e inacusativo), el tipo de construcción sintáctica o fuerza ilocutiva (interrogativa o exclamativa), así como las funciones informativas (Fernández Soriano 1993, Zubizarreta 1999, Silva-Corvalán 1982, 1984, Suñer 1982, MeyerHermann 1990). También ha recibido cierta atención la variación en la posición del sujeto pronominal, desde el punto de vista de la preminencia cognitiva y del estilo comunicativo (Serrano 2012, 2014). Los estudios que han tratado el tema, por tanto, han puesto de manifiesto y han dado cuenta de la complejidad del fenómeno y, por esta misma razón, no se ha encontrado una respuesta única a la variación sintáctica, que agote las posibilidades de análisis y explicación.

Raquel Hidalgo Downing, Universidad Complutense de Madrid, Madrid, España 
En relación con la investigación que se propone aquí, son particularmente relevantes, sin embargo, los trabajos con enfoque funcional y vocación discursiva, como Suñer (1982), Bentivoglio (1983), y Bentivoglio y Weber (1986), quienes trazaron unas tendencias básicas en el español, que posteriormente han recibido continuación en estudios de carácter cuantitativo o de revisión, y que estudian el comportamiento discursivo del sujeto (Givón 1990). Así pues, factores pragmáticos como la distribución de la información, la construcción tética o temática, presentativa, como la denomina Suñer (1982), arrojan luz sobre la naturaleza semántica y la funcionalidad discursiva de las distintas posiciones del sujeto en español. No obstante, los resultados no siempre han sido coincidentes, como señala Meyer-Handmann (1990), quien compara los distintos estudios en virtud de la metodología empleada, cuantitativa o cualitativa, así como el tipo de datos.

No es el propósito de este trabajo revisar con exhaustividad toda la tradición de estudios y posturas relativas a la posición del sujeto en español, como tampoco rebatirlas o invalidarlas, sino proponer un modelo de análisis de las funciones pragmáticas que pueda ser aplicado al estudio de textos, y que permita poner al descubierto y comprender el comportamiento pragmático-discursivo de los sujetos en español, a partir de la observación de la posición preverbal y posverbal en sus contextos de aparición en el discurso. De esta manera, se propone estudiar la posición del sujeto en relación con la estructura temática del discurso, lo que podría contribuir a mostrar cuál es la motivación y la funcionalidad de estas posiciones en la organización discursiva.

Para ello, se propone un modelo de análisis fundamentado en la descripción detallada de la función pragmática tema-tópico, y su análisis en el discurso. Con este fin, se ha recogido un cuerpo de datos formado por 10 textos, escritos y orales, procedentes de distintos géneros discursivos, y sobre los que se realiza un análisis empírico, atendiendo a la función tema y sus subdivisiones en tema nuevo, tema dado, tema subordinado y tema reasuntivo. Los resultados del análisis permitirán determinar que el sujeto en español presenta funciones temáticas distintas en las dos posiciones sintácticas principales, preverbal y posverbal. Mientras que el sujeto en posición preverbal introduce temas dados y conocidos y articula la continuidad temática, la posición posverbal introduce temas nuevos en el discurso.

El artículo se organiza de la manera siguiente: en la sección 2, se describe el modelo de descripción de las funciones pragmáticas; en la sección 3, se presentan los datos y la metodología de trabajo y en la sección 4 se presentan y discuten los resultados del estudio, en el que examinaremos sobre los textos las posiciones del sujeto y las características pragmático-discursivas de tales apariciones. El artículo se cierra con las conclusiones y la bibliografía. 


\section{El estudio de las funciones pragmáticas en la gramática y el discurso}

A pesar de las diferencias en la descripción de las funciones pragmáticas y su manifestación en las lenguas, algunos conceptos se emplean de forma recurrente y producen consenso en la medida en que plantean nociones que contribuyen a describir el orden de los constituyentes en función del contexto y el cotexto. Tanto la noción de tema (o tema-tópico) como su dimensión discursiva y textual (topicality) trazan las funciones pragmáticas que articulan la estructura informativa del discurso.

$\mathrm{Si}$ bien hay divergencias en los distintas enfoques y modelos teóricos, existe consenso en definir el tema-tópico como "aquello de lo que trata" el enunciado, siguiendo el uso común, intuitivo y pre-teórico del término tema como aquello de lo que trata un libro o una conversación:

The topic of a book, a film, etc. is taken to be what the book or film is about. So "topic of" expresses the relation of being about (Reinhart 1982, 1).

Topicality is a property of nominal participants of clauses. Propositional information, coded in state or event clauses, tends to be about some topical participant(s) in the state/ event (Givón 1990, 740).

The topic presents the entity about which the predication predicates something in the given setting (Dik 1989,16).

En los estudios especializados, se destaca asimismo el hecho de que el tema se articula en al menos tres niveles, el discurso, la secuencia y la oración, por lo que podemos decir que el tema puede ser: (a) el tema global o el tema del discurso; (b) el tema de un párrafo o una secuencia, y (c) el tema-tópico oracional, en relación con los temas de las oraciones precedentes (Reinhart 1982, van Dijk 1981).

Así pues, Dik $(1997,119)$ define el tema del discurso como "a topic expression whose referent is pragmatically salient beyond the limit of a single sentence”, es decir como el tema cuyo referente sobresale pragmáticamente más allá de una sola oración. La definición de Dik intenta captar la relación que existe entre los tres niveles, en la medida en que el tema se construye progresivamente: desde los temas oracionales, que configuran secuencias, a las que estas conforman remitiendo a un tema global o tema del discurso, que no puede comprenderse totalmente sino al final, cuando se ve en retrospectiva.

Los temas oracionales se identifican como entidades discursivas o participantes prominentes en el discurso: 
A discourse, taken in the wide sense of any kind of coherent text (a story, a monologue, a dialogue, a lecture etc.), is "about" certain entities. For example, this book is "about" Functional Grammar, this chapter is "about" pragmatic functions, and this subsection is "about" Topic and Topicality. For those entities about which a certain discourse imparts information we may use the term Discourse Topic (Dik 1997, 313-314).

El tema-tópico oracional está determinado por el discurso inmediatamente precedente en aquellos casos en los que existen varios candidatos posibles para acceder al tópico oracional. La elección de uno $\mathrm{u}$ otro depende de lo que podríamos denominar "tópico de la secuencia". El tópico de una secuencia es un tópico que permanece idéntico en una secuencia de oraciones (van Dijk 1981, 182). Un ejemplo es el fragmento ilustrado en (1):

(1) Esa mañana Eva se levantó a las cinco. Era el primer día de su nuevo trabajo en Praga. Se duchó rápidamente y desayunó. El tren salía a las seis y cuarto y no quería llegar con retraso en su primer día. Estaba demasiado nerviosa para leer el periódico en el tren.

"Eva" es el tópico de la secuencia ilustrada en (1), tal como demuestra la repetición del referente en las oraciones subsiguientes. Aun así, el tópico no debe identificarse con cualquier referente discursivo. Por ejemplo, aunque la expresión referencial "el tren" aparece dos veces en el texto, no diríamos que la secuencia "habla o trata del tren" en el sentido en que hemos discutido la noción intuitiva de tópico hasta ahora. Sin embargo, hay dos oraciones en las que "el tren" es el tópico oracional, en cuanto "dicen o predican algo del tren".

De esta discusión se deduce que la estructura tópica de la oración no depende solamente de la identidad referencial (o conectividad de las expresiones), sino también de la estructura tópica de la oración precedente, o incluso de una secuencia de oraciones (van Dijk 1981, 183).

El tercer nivel es el tópico del discurso. Ya se ha explicado que el tópico de una secuencia se define como la referencia repetida de un referente discursivo determinado, del cual los diferentes comentos de la secuencia aportan o especifican diversas propiedades o relaciones con otros, variables, referentes discursivos (van Dijk 1981, 186-87). En este caso, puede ocurrir que la secuencia contenga oraciones con otros tópicos oracionales, con los que el referente discursivo principal se relaciona; por ejemplo, en (1), "el tren". De la misma forma intuitiva que presentamos anteriormente, podemos decir que el tópico de discurso de (1) puede expresarse en (2): 
(2) Eva cogió el tren hacia Praga y empezó su nuevo trabajo.

(3) Esa mañana Eva se duchó y desayunó.

(4) Eva fue a Praga, pero no pudo leer el periódico en el tren.

Una oración como (2) puede aceptarse también como un resumen de la historia. Naturalmente, otros son posibles, pero serán variantes de (2), mientras que otros resúmenes serían claramente inadecuados como respuestas a la pregunta, ¿de qué trata la historia de (1)? Por ejemplo, la historia no es sobre el hecho de que Eva se duchó esa mañana, como sugeriría (3), o sobre la lectura del periódico (4). En otras palabras, el tópico está determinado por lo que, desde una cierta perspectiva, conforma los hechos más importantes de la historia. El resumen de (2) no aparece textualmente en (1), sino que es un "constructo", que se ha creado uniendo información que aparece en el discurso, como un todo.

No obstante, el tema no se manifiesta necesariamente de forma explícita en la lengua, por lo que el analista puede encontrar serias dificultades en identificar de forma inequívoca el tema en los tres niveles. La aparición de determinados indicadores, como los títulos, subtítulos, párrafos, imágenes o expresiones metadiscursivas (Cortés Rodríguez e Hidalgo Downing 2015), pueden servir de orientación al oyente/lector acerca de la organización temática del texto, pero estos mecanismos no están siempre presentes, y no son exclusivos de la articulación temática, sino que desempeñan otras funciones lingüísticas.

\subsection{La subdivisión en tipos de Tema}

El tema se articula en el discurso, estableciendo relaciones entre unos referentes discursivos y otros y mediante dos movimientos básicos o principales, como son el cambio temático y la continuidad. Para poder comprender y observar esta articulación, es preciso considerar la distinción entre distintos tipos temáticos, que reflejen de un modo sutil la organización discursiva. Dik (1989, 1997) propone varios tipos, como son: Tema Nuevo, Tema Dado, Tema Subordinado y Tema Reasuntivo (New Topic, Given Topic, Sub Topic y Resumed Topic). La propuesta de Dik permite estudiar la organización temática del texto en una serie de categorías que reflejan movimientos temáticos principales: la continuidad temática (Tema Dado) y el cambio temático (Tema Nuevo), así como la progresión temática (Tema Subordinado) y la reintroducción temática (Tema Reasuntivo). Las categorías de Dik se relacionan con el valor informativo de los referentes discursivos, pero no los reflejan directamente, y no los reflejan 
todos. El valor informativo se aplica a todos los referentes discursivos, sean temas o no, mientras que las categorías de Dik recogen únicamente aquellos referentes que desempeñan funciones temáticas. En una oración, hay un solo tema o bien ninguno (juicios téticos o sin tema). En cambio, hay tantos referentes discursivos como participantes en el discurso (SSNN y pronombres).

Se define el Tema Nuevo como aquel que se introduce por primera vez en el discurso (Dik 1997, 314); en las lenguas aparece de forma recurrente una serie de construcciones sintácticas para introducir nuevos temas en el discurso, como los SSNN léxicos con nombres propios, posiciones posverbales, construcciones presentativas, indicadores y expresiones metalingüísticas que anuncian el cambio temático y la introducción del nuevo asunto en el discurso (Cortés Rodríguez e Hidalgo Downing 2015):

(5) Te voy a contar una historia sobre un elefante llamado Dumbo (Dik 1997, 315, mi traducción)

La diferencia entre enunciados sin tema y el Tema Nuevo constituye un fenómeno complejo y sobre el que no hay consenso. Para algunos lingüistas, la oración de (5) sería una oración sin tema, puesto que un constituyente no puede ser temático si no ha sido previamente introducido como tal. Para Dik, en cambio, un constituyente puede ser Tema Nuevo, como en (5), en aquellas construcciones que responden precisamente a la función de introducir ese constituyente como Tema.

Siguiendo la idea de Dik, desde mi punto de vista, un constituyente es Tema Nuevo cuando su función es la de introducir un referente en el discurso, que habrá de desempeñar un papel como participante del discurso, y por tanto puede ser tema oracional en la oración $\mathrm{u}$ oraciones subsiguientes. Una continuación de (5) sería en este caso (6):

(6) Dumbo era joven y tenía unas orejas grandes y características.

Un enunciado sin tema, en cambio, presenta un acontecimiento como un todo, como un evento, y los referentes discursivos no tienen por qué presentar participantes que jugarán un papel en la historia o el discurso. En ese caso tendríamos (7):

\section{(7) Era un día soleado en la sabana.}

A diferencia de (5), el enunciado de (7) presenta un evento que aporta información de fondo sobre la historia, y "un día soleado" no introduce un 
referente discursivo que jugará un papel como participante. Se pueden emplear los criterios siguientes para distinguir los enunciados sin tema de los enunciados que introducen un Tema Nuevo (siempre en la clasificación de Dik 1997):

(i) Se puede añadir al enunciado un indicador temático, como "en cuanto a", “en relación a”;

(ii) El constituyente puede ser un participante en el discurso, y no simplemente información de fondo;

(iii) El referente discursivo aparece como tema oracional en las oraciones siguientes.

Mientras que los criterios se cumplen en (5), no se cumplen para (7).

La segunda categoría es el Tema Dado, que se refiere a un tema que ya se ha establecido en el discurso. Una vez que se ha introducido un Tema Nuevo, este pasa a Tema Dado en las oraciones siguientes. Este tipo temático refleja las estrategias y mecanismos de mantenimiento del tema que se conocen en la bibliografía también como tópicos continuos (Givón 1983, Goutsos 1997) y se marcan mediante mecanismos como la anáfora, la correferencia, la concordancia verbal o anáfora cero y la repetición léxica.

Los hablantes emplean algunas de estas estrategias para mantener el tema siempre y cuando sea pertinente en la comunicación.

(8) Ayer recibí una llamada del inspector de Hacienda (Tema Nuevo). El hombre (Tema Dado) me solicitó que acudiera a la oficina de Hacienda, y ( $\emptyset$, Tema Dado) me dio a entender que había problemas con mis impuestos.

El tercer tipo, el Tema Subordinado, es un tema que puede inferirse de un Tema Dado a partir del conocimiento que tenemos del mundo: "a Topic which may be legitimately inferred from a GivTop on the basis of our knowledge of what is normally the case in the world" (Dik 1997, 324):

(9) Juan celebró una fiesta el viernes pasado, pero la música era horrible.

En (9), el conocimiento del mundo nos permite saber que la música es un elemento típico de una fiesta, y por tanto puede aparecer como si ya hubiera sido introducido en el discurso (Dik 1997, 323).

Por último, cuando un referente ha sido introducido en el discurso, pero no se ha mencionado recientemente, puede ser reactivado o reestablecido como un Tema Reasuntivo. Esto ocurre especialmente cuando en el discurso hay varios referentes ( $\mathrm{A}$ y $\mathrm{B}$, por ejemplo), y la conversación ha versado varias 
secuencias sobre el referente A. Para volver a tratar sobre B, resulta necesario emplear alguna estrategia de restablecimiento o restauración temática, tal como los marcadores de cambio temático:

(10) Juan tenía un hermano llamado Pedro y una hermana llamada María. [secuencia sobre Pedro]. En cuanto a María, la hermana de Juan que mencioné anteriormente, vivió en Australia durante mucho tiempo (Dik 1997, 325, mi traducción).

La estructura temática y el estatuto informativo de los referentes discursivos son dimensiones interdependientes, que guardan una interesante relación, si bien al mismo tiempo constituyen dimensiones diferenciadas. El valor informativo puede aplicarse a todos los referentes discursivos, mientras que las subdivisiones del Tema que propone Dik, Tema Nuevo, Tema Dado, Tema Subordinado y Tema Reasuntivo, se refieren únicamente a los constituyentes que desempeñan la función de tema-tópico en la oración, y no al resto de referentes discursivos.

Desde nuestro punto de vista y esta propuesta de análisis, las subdivisiones temáticas resultan de gran utilidad en el análisis de los textos porque permiten observar y describir el modo en que el Tema del Discurso se articula a través del discurso, desde el nivel del enunciado a la secuencia, y fundamentalmente con una visión prospectiva, es decir mirando hacia adelante. En este sentido, resulta interesante aplicar las subdivisiones del tema para comprender y captar los movimientos temáticos que articulan y organizan el discurso. Pueden distinguirse dos movimientos temáticos principales: la continuidad temática (Tema Dado) y el cambio temático. El cambio temático puede manifestarse a través de la introducción de un tema nuevo (Tema Nuevo), pero con frecuencia no es realmente así, sino que sucede a través de la progresión temática, es decir de la introducción de temas relacionados con temas anteriores (Tema Subordinado) o con temas anteriores pero relegados temporalmente (Tema Reasuntivo).

\section{Metodología y datos para la investigación}

Para el estudio de las posiciones del sujeto en español y su relación con la organización temática del texto, se ha recogido un conjunto de textos, escritos y orales, que presenta cierta variedad, y que permite observar la aparición de la posición preverbal y posverbal del sujeto. Entre los escritos, se ha recogido textos de carácter narrativo (cuentos, biografías y noticias) y expositivo. Entre los textos orales, se ha recogido entrevistas, discursos y conversaciones 
cotidianas. ${ }^{1}$ Son estas últimas dos conversaciones espontáneas coloquiales grabadas y transcritas de acuerdo con las convenciones del grupo Valesco. Mientras que las conversaciones son un tipo de discurso no planificado, la entrevista representa un discurso intermedio, en cuanto ha sido preparado (por el entrevistador, para formular las preguntas, y por el entrevistado, para contestarlas) y posee, por tanto, una estructura fija, aunque la actuación no está desprovista de cierta espontaneidad. En cuanto a los discursos políticos, en cambio, son ejemplos de discurso oral planificado, que ha sido preparado previamente (por escrito, sin duda) y que es de carácter fundamentalmente expositivo, o expositivo-argumentativo. En la Tabla 1 puede observarse la relación de datos recogidos para el estudio.

Tabla 1: Muestra de datos del estudio. Textos orales y escritos.

\begin{tabular}{|c|c|c|c|c|}
\hline Título del texto & Escrito/Oral & Género & Palabras & Oraciones \\
\hline 1. Pulgarcita & Escrito & Cuento infantil & 326 & 46 \\
\hline 2. Tomás Bata & Escrito & Obituario & 376 & 30 \\
\hline 3. Velázquez & Escrito & Biografía & 544 & 33 \\
\hline $\begin{array}{l}\text { 4. Siniestro } \\
\text { eléctrico }\end{array}$ & Escrito & Noticia & 634 & 43 \\
\hline 5. Guerra Civil & Escrito & Testimonio & 938 & 79 \\
\hline $\begin{array}{l}\text { 6. Entrevista } \\
\text { P. Sánchez }\end{array}$ & Oral & Entrevista & 4515 & 224 \\
\hline 7. Discurso Rey & Oral & Discurso & 1271 & 98 \\
\hline $\begin{array}{l}\text { 8. Discurso } \\
\text { Presidente }\end{array}$ & Oral & Discurso & 9560 & 534 \\
\hline 9. Conversación 1 & Oral & $\begin{array}{l}\text { Conversación } \\
\text { cotidiana }\end{array}$ & 284 & 20 \\
\hline 10. Conversación 2 & Oral & $\begin{array}{l}\text { Conversación } \\
\text { cotidiana }\end{array}$ & 735 & 41 \\
\hline Totales & & & 19183 & 1148 \\
\hline
\end{tabular}

El objetivo de la recopilación de la muestra es observar las apariciones del sujeto en español en textos escritos y orales y con características distintas, para que los

1 El conjunto de textos orales comprende una entrevista televisada con el político español Pedro Sánchez, emitida el día 2 de noviembre de 2015, en RTVE, realizada por la periodista Ana Blanco; el Discurso del Rey Felipe VI pronunciado la noche del 24 de diciembre de 2017 (el discurso conocido como Discurso de Navidad del Rey), el Discurso inicial del Debate del Estado de la Nación, del Presidente del Gobierno español (con fecha junio 2016), y dos conversaciones cotidianas, extraídas del Corpus Valesco. El corpus Valesco está disponible en el siguiente enlace: http://www.valesco.es/?q=es/corpus. 
contextos de aparición no estén directamente condicionados por el tipo de discurso, como ha ocurrido en estudios anteriores sobre el tema, así como poder ver, si es el caso, diferencias entre unos y otros. Naturalmente, la muestra no es grande, puesto que el modelo de análisis que se propone realizar sobre los textos exige un nivel elevado de detalle; por tanto, no pretende arrojar generalizaciones a gran escala, pero sí servir como referencia futura para realizar estudios más amplios, con mayor cantidad de datos, o como punto de partida para un estudio más profundo sobre el comportamiento del sujeto en la lengua oral o escrita, en distintos tipos de discurso o considerando otras variables.

Para el análisis, se ha segmentado cada uno de los textos de la muestra en oraciones, para poder examinar los sujetos y la posición que ocupan en relación al verbo. A continuación, se ha analizado el tema-tópico de cada oración principal, y de las oraciones subordinadas con formas flexivas del verbo, así como las oraciones que carecen de tema, obteniendo por tanto los tipos de Tema Nuevo, Tema Dado, Tema Subordinado, Tema Reasuntivo y Sin Tema. Se ha incluido las oraciones con anáfora cero como casos de Tema Dado o continuidad temática, y se han contabilizado como sujetos preverbales.

El análisis de los temas-tópicos de cada oración se ha realizado siguiendo los criterios generales de Dik (1997), que se han aplicado al análisis de textos orales (Hidalgo Downing 2003, Cortés Rodríguez e Hidalgo Downing 2015) y escritos (Hidalgo Downing y Downing 2012). Algunos de estos criterios son los siguientes (Hidalgo Downing y Downing 2012, 200) ${ }^{2}$ :

1. Cada oración puede tener un tema-tópico, pero no más de uno.

2. Puede haber oraciones sin tópico.

3. El tópico coincide mayoritariamente con el sujeto sintáctico, pero puede también coincidir con un complemento verbal.

4. Se pueden emplear expresiones metadiscursivas como "en cuanto a", "en relación a”, "voy a hablar de" para comprobar si un referente discursivo determinado desempeña la función de tema-tópico. El uso de estas expresiones permite distinguir las oraciones sin tópico (orientadas al evento) de las oraciones con un tópico nuevo.

Tras la anotación de los textos con estas categorías, se han extraído las tablas de frecuencias y distribución de aparición de cada uno de ellos, para examinar

2 En Hidalgo Downing y Downing (2012) se propone un sistema de anotación básico (core annotation scheme) y avanzado (extended annotation scheme) para la función tópico y su aplicación al análisis de los textos. 
las funciones discursivas de los diferentes tipos temáticos en relación con la posición preverbal y posverbal del sujeto.

\section{Resultados y discusión}

En esta sección se muestran los resultados del análisis y se ofrece una discusión sobre los valores encontrados y su interpretación. En la Tabla 2 se refleja la distribución general de los sujetos en posición preverbal y posverbal, en cada uno de los textos analizados.

Tabla 2: Posición del sujeto en los textos escritos y orales.

\begin{tabular}{|c|c|c|c|c|c|c|}
\hline \multirow[t]{2}{*}{ Texto } & \multicolumn{2}{|c|}{ Sujeto Preverbal } & \multicolumn{2}{|c|}{ Sujeto Posverbal } & \multicolumn{2}{|c|}{ Total } \\
\hline & № & $\%$ & № & $\%$ & № & $\%$ \\
\hline Pulgarcita & 36 & 78,26 & 10 & 21,74 & 46 & 100 \\
\hline Tomás Bata & 25 & 89,28 & 3 & 10,72 & 28 & 100 \\
\hline Velázquez & 29 & 93,54 & 2 & 6,45 & 31 & 100 \\
\hline Siniestro eléctrico & 36 & 78,26 & 10 & 21,74 & 47 & 100 \\
\hline Testimonio Guerra Civil & 45 & 88,00 & 6 & 12,00 & 52 & 100 \\
\hline Subtotal textos escritos & 171 & & 31 & & 204 & \\
\hline Entrevista con P. Sánchez & 206 & 94,00 & 12 & 6,00 & 218 & 100 \\
\hline Discurso del Rey & 88 & 98,00 & 2 & 2,00 & 90 & 100 \\
\hline Discurso Presidente & 462 & 94,00 & 28 & 6,00 & 490 & 100 \\
\hline Conversación 1 & 20 & 100,00 & 0 & 0,00 & 20 & 100 \\
\hline Conversación 2 & 39 & 95,00 & 2 & 5,00 & 41 & 100 \\
\hline Subtotal textos orales & 815 & & 44 & & 859 & \\
\hline
\end{tabular}

El dato general es la preferencia en todos los textos por la posición preverbal del sujeto, que es mayoritaria, puesto que supera o el $75 \%$ en todos los casos, lo que hace pensar que es la posición preferida en los textos tanto escritos como orales. ${ }^{3}$ Además, en todos los casos los porcentajes son claros y alcanzan

3 De los sujetos preverbales, una parte importante lo constituyen sujetos en anáfora cero, que desempeñan funciones de Temas Datos, y que suman un 60\% de los Temas Dados en los textos escritos, y un $75 \%$ en los textos orales. Este dato sugiere que la continuidad temática es un mecanismo fundamental en la construcción discursiva y en la asignación de las funciones pragmáticas. 
hasta el $100 \%$ en uno de los textos, la Conversación 1, en la que no aparece ningún sujeto posverbal. En otros se sitúa en el 98\%, del Discurso del Rey, un porcentaje también muy elevado, o el 94\% de la entrevista y el Discurso del Presidente. En los textos escritos, los porcentajes descienden, hasta el $78 \%$ en la historia infantil, aunque se mantienen mayoritarios.

De forma inversa, los sujetos posverbales son minoría en todos los textos; llegan a alcanzar el $21,74 \%$ en dos textos escritos, en su porcentaje más elevado, mientras que descienden notablemente en las conversaciones espontáneas, hasta un $5 \%$.

En los textos analizados, además, la distinción entre textos escritos y orales es relevante, ya que los textos escritos manifiestan una mayor presencia de sujetos posverbales, mientras que en los textos orales se produce de forma mayoritaria la construcción con el sujeto preverbal. Para poder dilucidar esta diferencia de comportamiento, hemos de analizar los tipos temáticos desde un análisis cualitativo y en los dos tipos de textos.

\subsection{Posición y funciones discursivas de los sujetos en textos escritos}

En la Tabla 3 se han contabilizado los sujetos en posición preverbal y posverbal de los textos escritos, en cada uno de los tipos de temas: Tema Nuevo, Tema Dado, Tema Sub, Tema Res, Sin Tema.

Tabla 3: Distribución de sujetos en posición preverbal y posverbal por subdivisión temática en textos escritos.

\begin{tabular}{|c|c|c|c|c|c|c|c|c|c|c|c|c|}
\hline \multirow[t]{2}{*}{$\begin{array}{l}\text { Posición } \\
\text { sujeto }\end{array}$} & \multicolumn{2}{|c|}{$\begin{array}{c}\text { Tema } \\
\text { Nuevo }\end{array}$} & \multicolumn{2}{|c|}{$\begin{array}{l}\text { Tema } \\
\text { Dado }\end{array}$} & \multicolumn{2}{|c|}{$\begin{array}{l}\text { Tema } \\
\text { Sub }\end{array}$} & \multicolumn{2}{|c|}{$\begin{array}{c}\text { Tema } \\
\text { Res }\end{array}$} & \multicolumn{2}{|c|}{$\begin{array}{l}\text { Sin } \\
\text { Tema }\end{array}$} & \multicolumn{2}{|c|}{ Total } \\
\hline & № & $\%$ & № & $\%$ & $\underline{0}$ & $\%$ & № & $\%$ & № & $\%$ & № & $\%$ \\
\hline & $\sigma$ & 3,46 & 107 & 60,69 & 27 & 15,60 & 34 & 19,65 & 1 & 0,60 & 175 & 100 \\
\hline Posverbal & 6 & 19,35 & 2 & 6,45 & 7 & 22,58 & 1 & 3,22 & 15 & 48,40 & 31 & 100 \\
\hline
\end{tabular}

En los textos escritos, aparecen diferencias interesantes en la posición preverbal y posverbal de acuerdo con el tipo de enunciado, con tema (temático) o sin tema (tético), así como en los diferentes subtipos de temas. En primer lugar, en la posición preverbal destaca el elevado porcentaje del Tema Dado, que es el preferido sin duda en esta posición. Este se corresponde con un tema que ha sido 
introducido en el discurso y que se mantiene en varias oraciones, con algunos de los mecanismos típicos para esa función:

(11) Tomás Bata tomó las riendas de la compañía en 1932. El empresario tenía tan solo 18 años, y era un aprendiz.

Tal como puede observarse en (11), el tema oracional Tomás Bata introducido en el discurso, se mantiene en las oraciones siguientes, conformando una secuencia; la continuidad temática se expresa a través de mecanismos como la cohesión léxica (el empresario) o la concordancia verbal (anáfora cero, era un aprendiz).

El hecho de que el Tema Dado sea el tipo temático más frecuente en la posición preverbal, sugiere dos fenómenos: el sujeto preverbal se identifica de forma muy pronunciada con el tema oracional, y sirve fundamentalmente para consolidar y mantener el tema en secuencias temáticas, que a su vez construyen y remiten al tema del discurso. Los ejemplos de este principio son numerosos en todos los textos:

(12) Diego Rodríguez de Silva y Velázquez, pintor barroco español, Øj nació en Sevilla en 1599. A los once años Øj inicia su aprendizaje en el taller de Francisco Pacheco, donde Øj permanecerá hasta 1617, cuando Øj ya es pintor independiente. Al año siguiente, con 19 años, Øj se casa con Juana Pacheco, hija de su maestro, hecho habitual en aquella época, con quien Øj tuvo dos hijas.

La biografía de Velázquez se inicia con un Tema Nuevo, marcado en el texto por el uso del nombre y los apellidos, y que presenta al personaje en el discurso por primera vez; en las oraciones siguientes, se emplea la anáfora, que instaura el mismo tema para toda la secuencia, mediante la continuidad temática. Estas construyen y remiten al tema del discurso. Tal como mostraba van Dijk (1981), no existe una relación directa entre el tema oracional y el tema del discurso, ya que este último es un constructo, una representación retrospectiva de lo que ha tratado el texto. Sin embargo, indirectamente sí existe una relación, en la medida en la que el texto muestra relaciones de conectividad (los temas oracionales se suceden y la continuidad temática permite el desarrollo de un tema) y al mismo tiempo de coherencia (el tema oracional remite a algún elemento o varios del Tema del discurso). Sin embargo, no todos los temas oracionales remiten al tema discursivo.

El segundo tipo que aparece en posición preverbal es el Tema Reasuntivo; cuando un referente ha sido introducido en el discurso, pero ha sido relegado temporalmente, precisa de algún indicador que lo restaure: lo devuelva al 
primer plano discursivo. En cuanto a la organización temática, se corresponde con la reintroducción o recuperación temática:

(13) Las llamas se extendieron tan rápidamente por la habitación y generaron una intensa humareda que, a través del pasillo, alcanzó al resto de las habitaciones, sobre todo a aquellas cuyas puertas habian sido abiertas por los ancianos en un intento de huir. La humareda fue tan intensa que, para evitarla, los residentes prácticamente hubieran tenido que arrastrarse por el suelo.

En (13), se introduce el referente discursivo una intensa humareda como nuevo Tema inicialmente, y se convierte en tema de varias oraciones subsiguientes, creando continuidad temática, que queda truncada por el cambio de referencia introducida por la oración de relativo cuyas puertas. Para volver a restaurar la humareda como tema, el autor emplea el SN léxico, mecanismo que dota de prominencia al tema. El mismo fenómeno se observa en (14):

(14) Tomás Bata era uno de esos empresarios que dejan huella, nunca mejor dicho. El magnate de origen checo, heredero de la marca de zapatos que lleva el nombre de su familia, murió el pasado 1 de septiembre en un hospital de Toronto (Canadá) por causas que no se precisaron. La marca Bata es el símbolo de todo un imperio familiar. El negocio lo fundó su padre en 1894, en la ciudad de Zlin, en la antigua Checoslovaquia. Nacido en Praga un 17 de septiembre de 1914, Tomás Bata tomó las riendas de la compañía en 1932.

En el texto de (14), se puede observar claramente la articulación del tema. El primer párrafo introduce a Tomás Bata como tema oracional, que se mantiene con los mecanismos típicos de continuidad temática (cohesión léxica, anáfora). El siguiente párrafo, introduce el Tema Subordinado la marca Bata, que se refiere a la empresa y al negocio, no a la persona. Tras varias oraciones con este tema, el autor del texto quiere volver a traer al primer plano discursivo a Tomás Bata, la persona. Este es el Tema Reasuntivo, que se marca con el SN léxico (el nombre completo).

De nuevo, la restauración temática se pone de manifiesto aquí en el empleo del nombre completo, puesto que si bien el referente discursivo es conocido (dado) desde el punto de vista informativo, puesto que todo el texto trata de él, el autor se sirve de un mecanismo que le aporte de nuevo prominencia en el discurso.

El tercer tipo temático por orden de frecuencia de aparición en posición preverbal es el Tema Subordinado: se trata de un tema que está relacionado 
con un tema anterior, ya sea porque comparte la situación de comunicación (situacional), ya sea por inferencia (inferencial) de distinto tipo, como la relación de carácter metonímico.

Este tipo temático es frecuente porque se corresponde con un movimiento temático fundamental, la progresión temática:

(15) Bata dejó la presidencia de su imperio en manos de su hijo en 2001, pero nunca se apartó del imperio para el que trabajan 4.000 personas. La sede de la compañía se encuentra en Lausana, Suiza.

Como puede observarse en (15), tras dos oraciones con el tema oracional Bata, la tercera oración cambia de tema, si bien no se trata de la introducción de un tema nuevo sino relacionado, ya que la sede de la compañía se relaciona metonímicamente con el tema anterior. En una línea similar, en (16) el tema oracional el revestimiento del techo se entiende como una parte de la estancia:

(16) La estancia resultó afectada [por el fuego] hasta el punto de que lo único que quedó en pie fue el esqueleto de la cama. [...] Incluso el revestimiento del techo se vino abajo.

Se trata de un mecanismo natural que permite desarrollar el Tema del Discurso a través de temas relacionados entre sí, que no constituyen estrictamente un cambio temático, pero tampoco expresan continuidad, sino que lo complejizan, aportando nuevas entidades discursivas.

La introducción de un Tema Nuevo, por otro lado, supone un mecanismo que se produce en menor medida en posición preverbal, pero también aparece, hasta en un 3,46\% de los datos, y se produce típicamente al inicio de los textos, cuando se introduce el referente que será Tema del Discurso (17) y (18), aunque también puede aparecer en posiciones distintas a las de inicio del texto (19):

(17) Tomás Bata era uno de esos empresarios que dejan huella.

(18) Diego Rodríguez de Silva y Velázquez, pintor español barroco, nació en Sevilla en 1599.

(19) Mi madre supo que su hermano pequeño estaba prisionero en un sitio de Marruecos. Entonces se acordó de mi tía Rosalía, que se fue de ama de leche a Casablanca. Rosalía y Adelaida eran hermanas de mi abuelo Paco, el padre de mi padre. Una de las dos, cuando estaba criando un niño, era ama de leche con una mujer rica de Casablanca, que no tenía leche para 
criar a su hijo. Cuando la señora se fue para Marruecos, se fueron las dos hermanas de Paco y sus familias con ella, y allí les buscaron colocación.

En (17) y (18) los nombres propios se emplean para introducir un Tema del Discurso que resulta totalmente nuevo también desde el punto de vista informativo, ya que aparece al inicio del texto. En (19), por su parte, Rosalía y Adelaida constituye un Tema Nuevo, que se introduce en esa oración y se mantiene durante varias oraciones subsiguientes.

Como una primera conclusión, por tanto, es destacable la distribución de los tipos temáticos en posición preverbal, puesto que reflejan los principios funcionales de construcción discursiva, según los cuales los sujetos preverbales sirven principalmente para indicar los movimientos temáticos de continuidad, recuperación y progresión, asegurando por tanto un papel clave en la articulación temática del discurso.

En la posición posverbal, en cambio, la distribución de los tipos temáticos es complementaria a la anterior. La mayor aparición de sujetos posverbales se corresponde con enunciados no temáticos o téticos, hasta un porcentaje del 48,40\%. Son numerosos los ejemplos de este tipo de enunciados, con sujeto posverbal:

(20) Pulgarcita cuidó de ella día y noche, hasta que llegó la primavera.

(21) La mujer plantó el grano y en seguida brotó una flor.

(22) Aunque se barajan dos hipótesis, los investigadores creen que el fuego se originó en un fallo eléctrico.

(23) Cuando empezó la Guerra Civil, todos los días salían camiones con los presos.

En todos los casos, los sujetos posverbales no constituyen el tema sobre el que se predica algo, sino que el enunciado se entiende como un todo, como un acontecimiento. En algunos casos, puede plantearse la duda, entre enunciados sin tema y enunciados que introducen un Nuevo Tema. Si consideramos que el tema ha de observarse prospectivamente, es decir por el movimiento temático que realiza en el discurso subsiguiente, se puede examinar si el sujeto posverbal es introducido realmente como Nuevo Tema, o bien no tiene continuidad alguna en el discurso.

En (20), hasta que llegó la primavera se entiende como un acontecimiento y por tanto carece de tema. El carácter no temático del constituyente la primavera se puede probar aplicando determinados criterios, como los siguientes: (i) si se añade una expresión que marca inequívocamente el tema (como en cuanto $a$, en 
relación a), el enunciado no resulta adecuado: En cuanto a la primavera, llegó; (ii) la primavera no pasa a tema oracional, de hecho (iii) no representa un referente que posee relevancia en el discurso, y es probable que no vuelva a aparecer. Así pues, la primavera no constituye una entidad discursiva que se introduce en el discurso y es un participante del mismo, sino que carece de persistencia tópica (Givón 1983) y no vuelve a aparecer en el texto.

Por otro lado, la introducción de un Nuevo Tema constituye asimismo una de las funciones principales de los sujetos posverbales, puesto que suma un $20 \%$ de los casos.

La diferencia, por tanto, es que el Nuevo Tema tiene persistencia tópica y tendrá presencia en el discurso. En estos casos, el sujeto posverbal introduce un Nuevo Tema que se instaurará como Tema en al menos una oración subsiguiente:

(24) Le abrió la puerta una monitora que, muy nerviosa, le dijo que el incendio había empezado en la segunda planta.

(25) - ¡Zzzzzz! -zumbó un abejorro que cogió al vuelo a Pulgarcita.

(26) Muy a menudo las visitaba un topo vecino que, enamorado de la voz de Pulgarcita, se quería casar con ella.

Tal como se ha apuntado anteriormente, en (24) a (26) el sujeto posverbal introduce un Nuevo Tema que se instaura como tema en las oraciones siguientes; por tanto, esta construcción se entiende como un mecanismo típico de introducción temática en el discurso. Es este tipo temático el que se corresponde de un modo más claro con la función presentativa de los sujetos posverbales que han observado otros autores (Suñer 1982, Bentivoglio 1983).

Un grupo importante de sujetos posverbales, hasta un 22\%, lo constituyen los temas subordinados, que contribuyen a la progresión temática:

(27) En 1623 [Velázquez] se traslada a Madrid donde obtiene el título de Pintor del Rey Felipe. A partir de ese momento, empieza su ascenso en la Corte española, y realiza interesantes retratos.

(28) Al año siguiente, con 19 años, se casa con Juana Pacheco, hija de su maestro, hecho habitual en aquella época, con quien tendrá dos hijas. Entre 1617 y 1623, se desarrolla la etapa sevillana, caracterizada por el estilo tenebrista, influencia de Caravaggio, y que destaca con obras como El Aguador de Sevilla. 
En estos casos, los temas están relacionados con el tema principal del discurso (Velázquez) por relación metonímica, y destaca el hecho de que estos temas en posición posverbal se convierten en temas en las oraciones siguientes, articulando una pequeña secuencia del texto.

De forma mucho más limitada, casi reducida, se encuentran sujetos posverbales que indican Temas Dados, es decir continuidad temática:

(29) Los agentes tuvieron que retrasar la inspección ocular de la habitación de la segunda planta de la residencia en la que se inició el fuego, precisamente la que ocupaba una prima hermana de la propietaria del centro geriátrico, ya que la estancia resultó afectada hasta el punto de que lo único que quedó en pie era el esqueleto de la cama en la que pereció carbonizada la mujer. Incluso el revestimiento del techo se vino abajo. Aunque se barajan dos hipótesis, los investigadores creen casi con seguridad que el incendio se originó por un fallo en la corriente eléctrica a la que se conecta el colchón antiescaras (para evitar úlceras) en el que dormía la víctima.

En (29), el sujeto posverbal aparece en una oración subordinada que aporta información adicional que relaciona temáticamente el enunciado con los temas anteriores (la víctima, uno de los temas principales).

En conclusión, los sujetos posverbales se caracterizan por introducir fundamentalmente enunciados no temáticos, que contribuyen a la construcción del discurso porque aportan acontecimientos, circunstancias e información que actúa como marco para el desarrollo propiamente temático. Asimismo, introducen temas nuevos en el discurso, lo que equivaldría a la función presentativa señalada por otros autores (Suñer 1982, Bentivoglio 1983), y en una línea similar participan de la progresión temática. No se espera, en cambio, que actúen como marcas de continuidad temática, salvo en construcciones sintácticas específicas, tales como las subordinadas, que aportan información secundaria y que operan por tanto en el plano del transfondo discursivo, y no del primer plano.

\subsection{Posición y funciones discursivas de los sujetos en textos orales}

En los textos orales, la distribución de sujetos en posición preverbal y posverbal es similar a la de los textos escritos, con mayoría de sujetos en posición preverbal para los Temas Dados, y la aparición de los Temas Nuevos 
Tabla 4: Distribución de sujetos en posición preverbal y posverbal por subdivisión temática en textos orales.

\begin{tabular}{|c|c|c|c|c|c|c|c|c|c|c|c|c|}
\hline \multirow[t]{2}{*}{$\begin{array}{l}\text { Posición } \\
\text { sujeto }\end{array}$} & \multicolumn{2}{|c|}{ Tema Nuevo } & \multicolumn{2}{|c|}{$\begin{array}{l}\text { Tema } \\
\text { Dado }\end{array}$} & \multicolumn{2}{|c|}{$\begin{array}{c}\text { Tema } \\
\text { Sub }\end{array}$} & \multicolumn{2}{|c|}{$\begin{array}{c}\text { Tema } \\
\text { Res }\end{array}$} & \multicolumn{2}{|c|}{$\begin{array}{l}\text { Sin } \\
\text { Tema }\end{array}$} & \multicolumn{2}{|c|}{ Total } \\
\hline & № & $\%$ & № & $\%$ & № & $\%$ & № & $\%$ & № & $\%$ & № & $\%$ \\
\hline Preverbal & 0 & 0,00 & 624 & 73,50 & 120 & 14,50 & 56 & 7,00 & 40 & 5,00 & 840 & 100 \\
\hline Posverbal & 5 & 26,50 & 2 & 10,50 & 1 & 50,00 & 0 & 0,00 & 11 & 58,00 & 19 & 100 \\
\hline
\end{tabular}

y enunciados no temáticos en la posición posverbal. Los resultados se muestran en la Tabla 4.

La diferencia fundamental con respecto a los textos escritos es la menor incidencia de los sujetos posverbales, cuya aparición es considerablemente menos frecuente, lo que sugiere una incidencia mayor de la continuidad temática y de la reutilización de información conocida en el discurso, así como de sus participantes, que constituyen temas del discurso. Así ocurre por ejemplo en la conversación espontánea, donde se pueden encontrar sujetos posverbales que corresponden a Temas Dados, con valor contrastivo:

(30) Es el único que cuando nadie se atreve, se atreve él, tío.

(31) Y va y lleva a un testigo la otra, que también es de La Fe y estaba a doscientos metros y no vio casi nada, nano.

En (30) el sujeto posverbal es efectivamente Tema Dado, porque es conocido para el oyente, pero se marca en posición posverbal con valor contrastivo. En (31), de forma similar, en las intervenciones anteriores los hablantes están discutiendo sobre un robo que sufrió un amigo cuando iba en una moto y el juicio que se desarrolló a continuación; la otra se refiere a la abogada del acusado, por tanto, un Tema Dado pues ya se ha hablado previamente de ella.

No obstante, al igual que ocurría con los textos escritos, la posición posverbal es particularmente rentable a la hora de introducir enunciados no temáticos o téticos, que marcan un acontecimiento como un todo y no como un tema y un comentario que predica algo sobre el tema:

(32) Los españoles deben decidir el 20 de diciembre. Las formaciones nuevas tienen mucho de viejos conocidos. Hoy por ejemplo en Madrid se han aprobado 
presupuestos autonómicos, recortando partidas de educación, y han dejado las partidas de educación privada. ${ }^{4}$

El entrevistado habla sobre los nuevos partidos políticos, tema del discurso que aparece en continuidad temática en la última oración (han dejado ... ). En la oración con sujeto posverbal, en cambio, no se introduce un referente nuevo en el discurso que pueda constituir un Tema, sino que se marca el acontecimiento; se expresa sintácticamente mediante una construcción con se; una configuración sintáctica que precisamente sirve para introducir acontecimientos que se perciben en su totalidad, y que llevan sujeto posverbal.

En otras ocasiones de la entrevista, el político emplea este tipo de construcción con propósito similar:

(33) En una opción de socialdemócratas, se han hecho grandes cosas por el país, han puesto en pie el Estado de Bienestar, se han aprobado las principales leyes de derechos y libertades. Si me pregunta con cuál formación política me siento más cerca, yo le digo que, con las derechas, yo no me siento unido.

Resulta interesante la articulación temática de esta intervención porque en ella puede verse la funcionalidad de las dos construcciones, preverbal y posverbal. Mientras que en una opción de socialdemócratas constituye el marco temático que introduce el tema discursivo, y de hecho se recupera anafóricamente en la oración subsiguiente (han puesto en pie el Estado de Bienestar), las dos oraciones con sujeto posverbal refieren los acontecimientos logrados en esa etapa política, si bien sintáctica y pragmáticamente se marcan como eventos no adscritos directamente al tema principal, lo que confiere a las construcciones un efecto generalizador, no limitado a la acción de una persona, o una estrategia de impersonalización o cortesía de alejamiento, o simplemente una relación de los acontecimientos logrados.

En los sujetos posverbales se han hecho grandes cosas por el país y se han aprobado leyes, el orador presenta estos hechos como un todo, no introduce referentes discursivos que en las oraciones subsiguientes se convertirán en temas oracionales. Este tipo de construcción posverbal no temática aparece en los textos orales formales o planificados con cierta frecuencia, ya que los oradores la emplean para introducir acontecimientos que han realizado (los políticos) pero

4 Entrevista de la periodista española Ana Blanco a Pedro Sánchez, emitida en RTVE el 5 de noviembre de 2015 y accesible en la web de RTVE, http://www.rtve.es/alacarta/videos/especia les-informativos/especial-informativo-entrevista-pedro-sanchez-tve/3344751/. 
que no se atribuyen de forma personal, como también a acontecimientos del ámbito general de la Historia:

(34) Nunca antes en la Historia de la Humanidad, se habian producido cambios tan grandes (Discurso del Rey Felipe VI, 24/12/2017).

La posición posverbal de Tema Nuevo, en cambio, sí introduce entidades nuevas en el discurso, y de hecho se marcan así mediante recursos como SSNN léxicos, nombres propios completos, apellidos, profesiones etc.:

(35) Quiero expresar mi pesar a las familias de las víctimas de los atentados en Afganistán. Como consecuencia de ellos han perdido la vida el sargento Manuel Argudin Perrino y la soldado Niyireth Pineda Marín.

Por tanto, en los textos orales puede advertirse un uso menor de los sujetos posverbales que en los textos escritos. Ello parece indicar que los autores de los textos escritos emplean distintos mecanismos lingüísticos, principalmente sintácticos, para articular la organización temática del texto. Por ejemplo, acuden a las construcciones no temáticas para introducir información de fondo y circunstancias de la historia, mientras que se sirven de la introducción de temas nuevos para presentar temas nuevos en el discurso. Además, una vez introducido un tema nuevo, los mecanismos de correferencia permiten constituir cadenas tópicas y crear cohesión. En los textos orales, los hablantes optan por la reutilización de los referentes discursivos en temas dados, conocidos, y emplean la posición posverbal con una frecuencia mucho menor. En esta posición posverbal, destaca la aparición de sujetos posverbales no temáticos, que aquí introducen acontecimientos o hechos a los que no quiere atribuirse una responsabilidad o que pertenecen al ámbito del acontecer general de la sociedad, y aparecen en el discurso político como una estrategia de despersonalización y distanciamiento discursivo. También en la posición posverbal se introducen temas nuevos, y sujetos con valor contrastivo.

\section{Conclusiones}

En este trabajo se ha propuesto un estudio de la posición del sujeto en español en relación con la función pragmática tema, con el propósito de investigar si el papel que juega el sujeto preverbal y posverbal en la organización discursiva y, en concreto, en la articulación temática del texto, es similar o bien puede 
diferenciarse claramente. Para ello, se ha propuesto un modelo de análisis que parte de la noción de tema como "aquello de lo que trata el enunciado", en la línea de las investigaciones que tratan el tema como una relación (aboutness) y no como una posición lineal. Además, se reconoce que la función pragmática opera al menos en tres niveles (oración, secuencia y discurso); si bien no se formula necesariamente de forma explícita en cada uno de ellos, ha de existir una relación de conectividad y coherencia entre ellos. Pero en el estudio se ha trabajado, principalmente, sobre la clasificación del tema-tópico en subtipos, siguiendo la clasificación de Dik: Tema Dado, Tema Subordinado, Tema Reasuntivo y Tema Nuevo. La subdivisión temática permite examinar los movimientos temáticos básicos (cambio y continuidad) con una apreciación más fina y detallada.

Una vez realizado el análisis de todos los sujetos de un conjunto de textos escritos y orales, se ha podido comprobar una serie de principios interesantes acerca de la influencia importantísima de la pragmática, en este caso del contexto, así como de los principios de construcción del discurso, sobre la elección de las construcciones sintácticas.

En primer lugar, se ha comprobado que la posición preverbal es mayoritaria en todos los textos, y además es una posición que muestra gran variedad de subtipos temáticos, con predominio sin embargo del Tema Dado, seguido por el Tema Reasuntivo y el Tema Subordinado. Ello indica que la posición preverbal recoge distintas funciones temáticas, pero sirve principalmente para articular los temas oracionales en secuencias y los proyecta sobre el discurso (Temas Dados), permitiendo que estos se mantengan y desarrollen (Temas Subordinados), en los mecanismos de progresión temática, y también sean recuperados y traídos de nuevo al primer plano discursivo (Tema Reasuntivo). En menor medida, los sujetos preverbales introducen Temas Nuevos en el discurso, normalmente con mecanismos como los nombres propios completos.

En cuanto a los sujetos posverbales, la función principal es introducir enunciados téticos o no temáticos, que expresan acontecimientos de forma global, sin predicar del tema. Esa sería, sin duda, la función principal de la posición posverbal, seguida de la introducción temática, es decir de Temas Nuevos en el discurso. Un modo de distinguir estas dos funciones consiste en observar la función prospectiva del sujeto, es decir si tras ser introducido en la posición posverbal, pasa a ser Tema de oraciones siguientes (introducción temática) o bien no tiene continuidad en el discurso (enunciado tético).

Por tanto, cabe destacar que, de acuerdo con el análisis de los textos recogidos, la posición del sujeto en español representa funciones distintas en la organización discursiva, y de hecho se encuentran casi en una distribución 
complementaria, puesto que se orientan a funciones inversas, con una mayor variedad en la posición preverbal, y una mayor especialización en la posverbal.

Asimismo, se han encontrado diferencias entre los textos escritos y los orales, puesto que en los primeros la aparición de sujetos posverbales es más elevada y más rica, mientras que se restringe más en el discurso oral, lo que sugiere que en este último los hablantes prefieren reutilizar la información y hacerla conocida al interlocutor. Sin embargo, algunos tipos de textos orales (los políticos), mostraron usos interesantes y distintos de la posición posverbal.

De todo ello se desprende que la posición del sujeto se relaciona con la organización temática del texto, y se pueden identificar las funciones temáticas principales para cada posición, aunque este estudio no ha determinado el impacto que puede tener el tipo de texto en el empleo de unos y otros tipos, que puede ser relevante.

\section{Referencias}

Bentivoglio, Paola. 1983. Topic continuity and discontinuity in discourse: a study of spoken Latin American Spanish. En Talmy Givón (ed.). Topic continuity in discourse: a quantitative cross-language study, 255-311. Amsterdam: John Benjamins.

Bentivoglio, Paola y Elisabeth C. Weber. 1986. A functional approach to subject Word order in spoken Spanish. En Osvaldo Jaeggli y Carmen Silva-Corvalán (eds.). Studies in Romance Linguistics, 23-40. Dordrecht: Foris.

Bernal, Josep María. 2007. El orden de palabras de la oración simple en español y en griego, RedELE, Revista Electrónica de didáctica y enseñanza de español lengua extranjera 9. $1-11$.

Cadiot, Pierre. 1992. Matching syntax and pragmatics: a typology of topic and topic-related constructions in spoken French. Linguistics 30. 57-88.

Chafe, Wallace. 1994. Discourse, Consciousness and Time. Chicago/London: The University of Chicago Press.

Contreras, Heles. 1978. El orden de palabras en español. Madrid: Cátedra.

Cortés Rodríguez, Luis y Raquel Hidalgo Downing. 2015. Indicadores de cambio temático en el discurso parlamentario: análisis de un discurso de Rodríguez Zapatero [2011]. Signos. Estudios de Lingüística 48(89). 279-306.

Dik, Simon. C. 1989. The Theory of Functional Grammar. Part I. Dordrecht: Foris.

Dik, Simon. C. 1997. The Theory of Functional Grammar. Part II. Dordrecht: Foris.

Dipper, Stephanie, Götze, Michael y Stavros Skopeteas. 2007. Information Structure in CrossLinguistic Corpora: Annotation Guidelines for Phonology, Morphology, Syntax, Semantics and Information Structure. Potsdam: Universitätsverlag Potsdam.

Fernández Soriano, Olga. 1993. Sobre el orden de palabras en español. Dicenda, Cuadernos de Filología Hispánica 11. 113-152.

Givón, T. 1983. Topic continuity in discourse: A quantitative cross-language study. Amsterdam: John Benjamins. 
Givón, Tom. 1990. Syntax. A Functional Typological Introduction. Volume II. Amsterdam: John Benjamins.

Goutsos, Dionysos. 1997. Modeling Discourse Topic: Sequential Relations and Strategies in Expository Text. Norwood: Ablex.

Gundel, Jeanette. 1985. Shared knowledge and topicality. Journal of Pragmatics 9 (1). 83-107. Hidalgo Downing, Raquel. 2003. La tematización en español hablado. Madrid: Gredos.

Hidalgo Downing, Raquel y Angela Downing. 2012. Topic and Topicality in Text: A contrastive study of English and Spanish narrative texts. Linguistics and the Human Sciences 6. 193-217.

Lambrecht, Knud. 1994. Information structure and sentence form. Topic, focus, and the mental representation of discourse referents. Cambridge: Cambridge University Press.

Maynard, Douglas W. 1980. Placement of topic changes in conversation. Semiotica 30. 3-4, 263-290.

Meyer-Hermann, Reynhard. 1990. Sobre algunas condiciones pragmáticas de la posición del sujeto en español. Estudios de Lingüística Universidad de Alicante 6. 73-88.

Morales de Walters, Amparo. 1982. La posición del sujeto en el español de Puerto Rico a la luz de la clase semántica verbal, la oposición tema-rema y el tópico oracional. Lingüística Española Actual IV. 23-38.

Morris, Thierry. 1988. Topicity vs. thematicity: topic prominence in impromptu Spanish discourse. Journal of pragmatics 29. 193-203.

Reinhart, Tanya. 1982. Pragmatics and linguistics: An analysis of sentence topics. Philosophica 27. 53-94.

Serrano, María José. 2014. Cognición y estilo comunicativo: el sujeto posverbal y el objeto sintáctico. Estudios Filológicos 54. 139-156.

Serrano, María José. 2012. El sujeto pronominal “usted/ustedes” y su posición: variación y creación de estilos comunicativos. Spanish in Context 9(1). 109-131.

Silva-Corvalán, Carmen. 1982. Subject expression and placement in Mexican-American Spanich. En Jon Amastae y Lucia Elías-Olivares (eds.). Spanish in the United States: Sociolinguistic Aspects, 93-120. New York: New York University Press.

Silva-Corvalán, Carmen. 1984. Topicalización y pragmática en español. Revista Española de Lingüística 14. 1-19.

Suñer, Margarita. 1982. Synatx and Semantics of Spanish Presentational Sentence-Types. Washington: G.U.P.

van Dijk, Teun. 1981. Sentence topic and discourse topic. En Studies in the pragmatics of discourse, 177-193. Paris: Mouton.

Zubizarreta, María Luisa. 1999. Las funciones informativas: tema y foco. En Ignacio Bosque y Violeta Demonte (eds) Gramática descriptiva de la lengua española, vol. 3, 4215-4244. Madrid: Real Academia Española/Espasa Calpe. 